Research Article

\title{
Forest Resource Use Pattern in Fringe Villages of Barsey Rhododendron Sanctuary and Singalila National Park of Khangchendzonga Landscape, India
}

\author{
Santosh K. Chettri $\mathbb{D}^{1,2}$ Ghanashyam Sharma, ${ }^{1}$ Kailash S. Gaira, ${ }^{2}$ Aseesh Pandey $\mathbb{D}^{2}{ }^{2}$ \\ Rajesh Joshi, ${ }^{2}$ Nakul Chettri, ${ }^{3}$ and Bharat Kumar Pradhan ${ }^{4}$ \\ ${ }^{1}$ The Mountain Institute (TMI)-India, Tadong, Gangtok, Sikkim, India \\ ${ }^{2}$ G. B. Pant National Institute of Himalayan Environment, Sikkim Regional Centre, Pangthang, Sikkim, India \\ ${ }^{3}$ International Centre for Integrated Mountain Development, Kathmandu, Nepal \\ ${ }^{4}$ Sikkim State Biodiversity Board, Forest Environment and Wildlife Management Department, Deorali, Gangtok, Sikkim, India \\ Correspondence should be addressed to Santosh K. Chettri; basnett66@rediffmail.com and Aseesh Pandey; \\ draseeshpandey@gmail.com
}

Received 15 September 2020; Revised 27 December 2020; Accepted 28 December 2020; Published 11 February 2021

Academic Editor: Daniel M. Kashian

Copyright $\odot 2021$ Santosh K. Chettri et al. This is an open access article distributed under the Creative Commons Attribution License, which permits unrestricted use, distribution, and reproduction in any medium, provided the original work is properly cited.

\begin{abstract}
Forests in the Barsey-Singalila transboundary area under Khangchendzonga landscape (KL) are facing pressures of resource uses. Despite continuous utilizations, complete data are lacking from the ground level. Socioeconomic, demographic, and forest use data were obtained by interviewing 233 households across two study locations. Forest product market survey was undertaken to determine prices of forest products. Resource dependence index was (0.60) high for both the locations. Alnus nepalensis is highly preferred fuel species with probability of use (0.791), and Rhododendron arboreum with probability of use (0.09) is considered as least preferred species. Relatively bigger village with more population is aggravating utilization pressures. Resource use at Gorkhey-Samanden is low ( $20 \%$ in case of fuelwood) as compared to the use at Ribdi-Bhareng ( $80 \%$ for fuelwood). Only preferred medicinal plants or wild edibles, namely, Yushania maling are collected regardless of their availability for fuelwood and other uses, which is causing more pressure on a small group of plants. A huge shortfall between resource demand and production indicates the possible extraction of resources from surrounding reserve forests. The results provide significant information on peoples dependency on forest resources and may be utilized for developing forest conservation policies for enhanced ecosystem services and livelihoods in the region.
\end{abstract}

\section{Introduction}

Forest is an essential component of various ecosystem services. Local people depend on forest resources for various products such as fuel wood, construction materials, medicine, and food [1]. Globally, it is estimated that between 1.1 billion and 1.7 billion people depend to varying degrees on forests for their livelihoods, and about 200 million indigenous communities are almost fully dependent on forests [2]. It is estimated that $20-25 \%$ of rural peoples' income is obtained from environmental resources in developing countries [3] and act as safety nets in periods of crisis or during seasonal food shortages $[4,5]$.
While in rural India, nearly 275 million people are directly dependent on forests for sustaining their livelihood [6]. Forest and people are inextricably linked in India, and around 350-400 million (40\%) people are dependent on forest resources [7]. About 70\% of Indian population lives in rural areas, and most of them have either agriculture or forest-based economy [8]. Their dependency on the forest resource is still high due to easy access, simple use, and lack of economically viable options [9]. Demand and consumption of fuelwood is not increasing over last decades as compared to the rate estimated earlier in 1980s, and it is still valid in rural areas [10]. The underutilized wild edible 
bioresources can play a significant role in rural development, poverty alleviation, livelihood enhancement, and nutritional security of local communities through bioprospecting with application of suitable scientific interventions [11-14].

Barsey-Singalila transboundary area within Khangchendzonga landscape (KL) houses unique ethnic and social groups with rich traditional knowledge on bioresource utilization [15]. Over the years, human pressure is exerted upon this transborder area for collection of basic subsistence materials, viz., fuelwood, fodder, construction timber, and nontimber forest produces (NTFPs). The dwelling community in the region employs different farming and livestock practices and also depends on forest resources for regular income generation. Hence, a comprehensive information on resource utilization patterns is required across state's border of KL. Also, to assess environmental impacts posed upon from the intervention is greatly sensed. Therefore, we planned to assess indigenous resource use patterns and people's dependency on bioresource needs. The study principally highlights on the resource availability, their use patterns, demand, and people's dependency on natural resources, for setting conservation priorities and livelihood security as apparent from the assessment.

\section{Materials and Methods}

2.1. Study Sites. Singalila National Park (SNP) having $78.6 \mathrm{~km}^{2}$ area is located on the Singalila Ridge at an altitude of $7000 \mathrm{~m}$ asl, in the Darjeeling district of West Bengal. It is famous for trekking route to Sandakphu that runs through it. The park was previously declared a wildlife sanctuary in 1986. The region had long been used as the trekking route from Manebhanjang to Sandakphu (the highest peak of West Bengal and Phalut). The trek along the Singalila Ridge to Sandakphu and Phalut is one of the most popular ones in the Eastern Himalaya, due to the grand vistas of the Khangchendzonga range, and the Everest range which can be seen from the ridge and also for the seasonal wildflower blooms and birding.

Barsey Rhododendron Sanctuary (BRS) covers an area of $104 \mathrm{~km}^{2}$, established in 2004 in the Singalila Ridge in western Sikkim. It borders on Nepal to the west and on the state of West Bengal to the south across the Rambong Khola stream. There are three points of entry to this sanctuary from Hilley, Dentam, and Soreng. Tourists generally prefer Hilley, since it is approachable by road too. The bridle path from Hilley to Barsey is a favorite amongst tourists especially during the Rhododendron flowering season. The faunal value of BRS includes leopard cat, Himalayan Yellow throated Marten, Himalayan Palm Civet, and many diverse species of birds. Two villages were selected for the present study from the Barsey-Singalila transboundary area, namely, Ribdi-Bhareng surrounding the BRS (Sikkim) and Gorkhey-Samanden (Darjeeling district, West Bengal) surrounding the SNP (Table 1).

Gorkhey-Samanden, a forest village located at an altitude of $2286 \mathrm{~m}$ asl, is the remotest village situated in the close proximity to the SNP (Darjeeling) in the north and BRS (Sikkim) in the east connected by an interstate border
(Figure 1). It is bestowed with rich biodiversity providing a wide range of ecosystem services and hence bears local significance. The village covers an area of 36 hectares. There are 65 households in the village with a total population of 205 persons comprising of Sherpa, Rai, Chettri, and Tamang communities. Male literacy rate of the village is $64 \%$; whereas, female literacy rate is $52 \%$. Agriculture, livestock, and tourism are major livelihood options of the community. Potato, maize, oat (barley), bean, rayosaag (green leafy vegetable), pea, cabbage, radish, and squash are common crops found. Apart from these, yacon (Smallanthus sonchifolius) is recently introduced speciesunder $\mathrm{KL}$ programme.

Ribdi-Bhareng, West Sikkim, is a Gram Panchayat Unit (GPU) with a total area of 543 hectares. There are 324 households in the GPU with total population of 1536 persons. Sherpa, Rai, Chettri, Tamang, and Gurung are the main dwelling communities. Male literacy rate of Ribdi-Bhareng is $60 \%$ and female 50\%. Agriculture and livestock rearing are major occupations of the people in Ribdi-Bhareng. Potato, maize, oat (barley), bean, rayosaag, pea, buckwheat, cabbage, cauliflower, radish, squash, and large cardamom are staple crops cultivated, and cattle are also kept for diary purposes.

\subsection{Methods}

2.2.1. Survey and Sampling. The socioeconomic survey of the households using semistructured questionnaire formats was carried out. A total of 233 households were surveyed from Gorkhey-Samanden (as site-A, $60 \mathrm{hh}$ ) and RibdiBhareng (as site- $\mathrm{B}, 173 \mathrm{hh}$ ). The data were collected through stratified random sampling [16] to select households in the sample villages to collect primary socioeconomic data on types of forest resource consumption, source, frequency, resource demand, and farm production. The forest product market survey was undertaken to determine prices of forest products. In addition, the PRA (including focus group discussions) survey was conducted. In the process, key stakeholders/informants, members of the farming community, ecotourism committee members, government employees, and the panchayat representatives were involved. Preferred fodder species were also documented from the households. Households were asked about the fuelwood, fodder, and other non-timber forest product collection and quantity of biomass that they extracted annually from the forest. Two individuals per household per day were found involved in collection of fuel. Weighed the head loads (bhari) for fuelwood at the entry and exit points of the forest boundary during November-March approximately for 150 days. Forty bharis were sampled in each village during each investigation. Collected amount of each species segregated out of each bundle based on local names and then weighed using a spring balance [17]. Living samples (twig containing leaf and flowers) of each species were locally identified and were later identified scientifically. To measure fuelwood utilization, actual number of hours burnt by a species was divided by the total number of hours that the fuel could have burnt [17]. Additional information were collected using field 
TABle 1: Detail of the study sites.

\begin{tabular}{lccccc}
\hline Study area & Latitude and longitude & $\begin{array}{c}\text { Total no. of } \\
\text { households }\end{array}$ & $\begin{array}{c}\text { Total } \\
\text { population }\end{array}$ & $\begin{array}{c}\text { Population involved for } \\
\text { collection }\end{array}$ & $\begin{array}{c}\text { No. of fuelwood species } \\
\text { available }\end{array}$ \\
\hline Ribdi Bhareng & $\begin{array}{l}27^{\circ} 9^{\prime} 32.14^{\prime \prime}-27^{\circ} 11^{\prime} 00.77^{\prime \prime} \mathrm{N} \\
88^{\circ} 4^{\prime} 37.29^{\prime \prime}-88^{\circ} 07^{\prime} 11.95^{\prime \prime} \mathrm{E}\end{array}$ & 324 & 1536 & 1396 & 32 \\
\hline $\begin{array}{l}\text { Gorkhey- } \\
\text { Samanden }\end{array}$ & $\begin{array}{l}27^{\circ} 10^{\prime} 39.26^{\prime \prime}-27^{\circ} 11^{\prime} 25.83^{\prime \prime} \mathrm{N} \\
88^{\circ} 04^{\prime} 02.24^{\prime \prime}-88^{\circ} 04^{\prime} 29.09^{\prime \prime} \mathrm{E}\end{array}$ & 65 & 205 & 178 & 31 \\
\hline
\end{tabular}

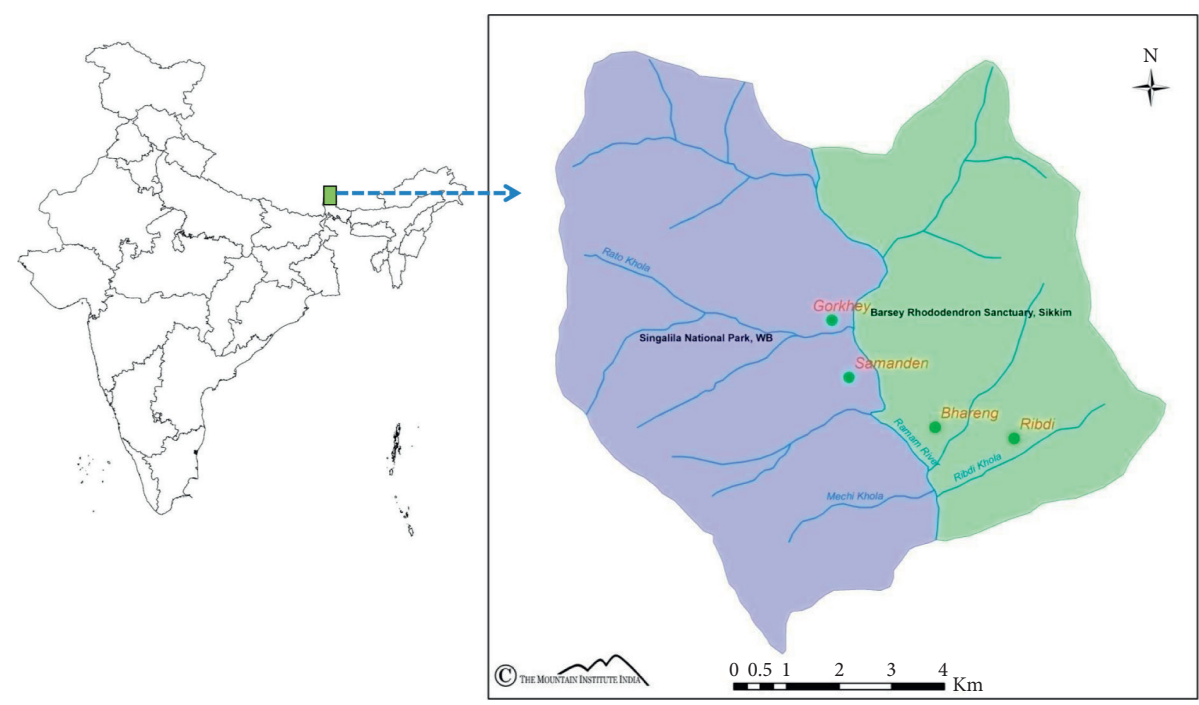

FIGURE 1: Map of the study area showing the location of study villages in Khangchendzonga landscape, India.

observations and transect walks. Information on price of different NTFPs/wild edibles and medicinal plants were collected through the market survey. The collected data on fuelwood were analysed following $[17,18]$.

\subsubsection{Data Analysis.}

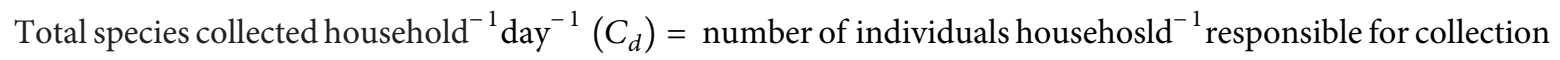

\&9; $\quad$ average collection $(A)$

or

$$
A=\frac{T}{N}
$$

where $T$ is the total species collected in all samples, and $N$ is the number of samples.

$$
\text { Probability of use }(\mathrm{PU})=\frac{\sum F_{i} P_{i}}{\sum P_{i}},
$$

where $F_{i}$ is the frequency of collection of a species in the $i^{\text {th }}$ site, and $P_{i}$ is the population of the $i^{\text {th }}$ site.

$$
\text { Resource use index }(\text { RUI })=\text { total species collection household } \text { year }^{-1} \times \text { PU }
$$

Fuelwood consumption among the sites was determined using Sorenson's similarity index [19]:

$$
\text { Similarity index }(\mathrm{SI})=\frac{2 C}{\mathrm{~A}+\mathrm{B}} \times 100 \text {, }
$$

where $C$ is the amount of fuel collected common in sites $(A$ and $B) ; A$ is the collection of fuel in site $A$, and $B$ is the collection of fuel in site $B$. Shortfall in resource needs and possible extraction are determined on the basis of the information collected. 


\section{Results}

3.1. Resource Availability Status and People's Dependency. With the aim of collecting data on resource base for villages, we carried out the seasonal study on resource extraction and utilization patterns. Results of the studied parameters depicted that fuelwood, fodder, timber, wild edibles, medicinal plants, and litter are the most common forest resources for Ribdi-Bhareng and Gorkhey-Samanden households (Table 2). Regardless of availability status, source of resource would be preferentially private forests (as rural people claim), reserved forest, national parks, and wildlife sanctuaries. We evidenced colloquial activities of tourism practices, forest collections, and grazing from the sites. People have to travel atleast 2-5 kilometers distances everyday to extract the resource. Wherein, the dependency index of studied sites was determined within 0.60 .

The fuelwood consumption at Ribdi-Bhareng was recorded slightly higher than Gorkhey-Samanden by $1.5 \%$; farmland production of fuelwood at Ribdi-Bhareng was more than private land fuelwood growth at GorkheySamanden by $80 \%$. This may be due to higher fraction of landholdings possessed by the house of Ribdi-Bhareng. The total number of households at Ribdi-Bhareng is 324 and Gorkhey-Samanden is 65. Likewise, fodder consumption rate was more at Ribdi-Bhareng (17\%) than fodder consumption at Gorkhey-Samanden, and in a similar way, the farm fodder production rate at Gorkhey-Samanden was lower than Ribdi-Bhareng (58.3\%).

Timber required at Gorkhey-Samanden was found 6 cubic $\mathrm{cm} \cdot \mathrm{hh}^{-1} \mathrm{yr}^{-1}$ which is much higher than the estimated production of 0.1 cubic $\mathrm{cm} \mathrm{hh}^{-1} \mathrm{y}^{-1}$. Similarly, timber demand in Ribdi-Bhareng was recorded 7.5 cubic cm $\mathrm{hh}^{-1} \cdot \mathrm{yr}^{-1}$, and the production was $0.2 \mathrm{cubic} \mathrm{cm} \cdot \mathrm{hh}^{-1} \cdot \mathrm{yr}^{-1}$. On the other hand, litter use at both Gorkhey-Samanden and Ribdi-Bhareng villages was found $10 \mathrm{~kg} \cdot \mathrm{hh}^{-1} \cdot \mathrm{d}^{-1}$ and $12 \mathrm{~kg} \cdot \mathrm{hh}^{-1} \cdot \mathrm{d}^{-1}$, respectively. Similarly, production amount was found $7 \mathrm{~kg} \cdot \mathrm{hh}^{-1} \cdot \mathrm{yr}^{-1}$ for both the villages. NTFP/wild edibles demand at Gorkhey-Samanden was $0.5 \mathrm{~kg} \cdot \mathrm{hh}^{-1} \cdot \mathrm{d}^{-1}$ and Ribdi-Bhareng $0.4 \mathrm{~kg} \cdot \mathrm{hh}^{-1} \cdot \mathrm{d}^{-1}$; whereas, production was found half of the amount, i.e., $0.25 \mathrm{~kg} \cdot \mathrm{hh}^{-1} \cdot \mathrm{d}^{-1}$ for both the above villages. Across the study sites, food stuffs (ration) required were estimated $12 \mathrm{~kg} \cdot \mathrm{hh}^{-1} \cdot \mathrm{month}^{-1}$ for a family having 6 members in each. Per capita crop production was estimated $3 \mathrm{~kg} \cdot \mathrm{month}^{-1}$ for a family.

3.2. Fuelwood Collection, Probability of Use (PU), and Resource Use Index (RUI). Habit and mean collection (kg sample ${ }^{-1} \cdot$ day $^{-1}$ and $\mathrm{kg} \mathrm{sample} \mathrm{e}^{-1} \cdot \mathrm{yr}^{-1}$ ) for common fuelwood species are presented in Table 3. Most species used for fuel are regarded as tree species. Mean collection for Arundo donax $\left(1.68 \mathrm{~kg} \cdot \mathrm{sample}^{-1} \cdot \mathrm{day}^{-1}\right)$ is found to be the most collected species followed by Quercus lamellosa $\left(1.62 \mathrm{~kg} \cdot \mathrm{sample}^{-1} \cdot \mathrm{day}^{-1}\right)$ and least for Tsuga dumosa and Ankhle $\left(0.11 \mathrm{~kg} \cdot \mathrm{sample}^{-1} \cdot \mathrm{day}^{-1}\right)$. For yearly extrapolation, Aundo donax $\left(252 \mathrm{~kg}\right.$.sample ${ }^{-1} \cdot$ year $\left.^{-1}\right)$ showed the most collected species followed by Quercus lamellosa Sm. (243 kg.sample ${ }^{-1} \cdot$ year $^{-1}$ ) and the least to Alnus nepalensis D.
Don (241.5 sample .year $\left.^{-1}\right)$. Probability of use (PU) is maximum for Arundo donax (0.813) followed by Alnus nepalensis (0.791), Dendrocalamus hamiltonii (0.745), and Castanopsis tribuloides (0.7) and least in Rhododendron arboreum (0.09).

Resource extraction processes were studied for fuel, fodder, timber, NTFPs/wild edibles, litter, and crop (Table 4). Our survey revealed that per year fuelwood consumption at RibdiBhareng is $\left(7409.5 \mathrm{kk} \cdot \mathrm{hh}^{-1}\right)$, fodder $\left(6570 \mathrm{kk} \cdot \mathrm{hh}^{-1}\right)$, and NTFPs $\left(146 \mathrm{~kg} \cdot \mathrm{hh}^{-1}\right)$ and Gorkhey-Samanden fuelwood is $\left(7300 \mathrm{kk} \cdot \mathrm{hh}^{-1}\right), \quad$ fodder $\quad\left(5475 \mathrm{~kg} \cdot \mathrm{hh}^{-1}\right)$, and NTFPs $\left(182.5 \mathrm{~kg} \cdot \mathrm{hh}^{-1}\right)$. Resource demand is compared with the resource production as shown in Table 5. Demand of fuelwood (2393.27 ton $\cdot \mathrm{yr}^{-1}$ ) and fodder (2122.11 ton $\cdot \mathrm{yr}^{-1}$ ) at RibdiBhareng was comparatively more than what is recorded from Gorkhey-Samanden (fuelwood $445.3 \mathrm{ton} \cdot \mathrm{yr}^{-1}$ and fodder 333.98 ton $\cdot \mathrm{yr}^{-1}$ ). Considering the fact that wood biomass is required for construction purposes such as house and goths/ cowshed, such is evident from timber requirement at RibdiBhareng (2422.5 cft/yr) and also production ( $64.6 \mathrm{cft} / \mathrm{yr})$ thereby leading to shortfall amount $(2357.9 \mathrm{cft} / \mathrm{yr})$. Fuelwood demand in Gorkhey-Samanden was $\left(445.3\right.$ ton $\left.\cdot \mathrm{yr}^{-1}\right)$, whereas production was $\left(0.12\right.$ ton $\left.\cdot \mathrm{yr}^{-1}\right)$. As per the primary survey of the villages, the fuel consumption was 418.86 ton, and the annual fuel availability was estimated as 211.03 ton, which indicates a deficit of 207.83 ton. Per family fuel consumption was found $52.53 \mathrm{~kg}$ which varies seasonally (summer, $17.28 \mathrm{~kg}$ and winter, $35.25 \mathrm{~kg}$ ).

3.3. Fodder Use. Farm animals in Barsey-Singalila are rearing by two means stall feeding and open grazing. Open grazing in forest sites has been banned in Sikkim under state's revised grazing policy, 2005 (remained effective from 2010), although illegal grazing inside forests occur. But the animal percentage meant for stall feeding is more (80\%). While studying the fodder preferences, 8 most preferred species, 12 preferred species, and 6 least preferred species were found for both Ribdi-Bhareng and Gorkhey-Samanden villages (Table 6).

3.4. NTFP Use. Varying degrees of NTFP use (as high, medium, and low) and availability status (abundant, moderate, and rare) were noted. People's dependency (high, medium, and low) and market prices of various wild edibles, NTFPs, and medicinal herbs were studied as shown in Table 7. It is observed that Diplazium sp. is priced to a low of only Rs. 10/bundle, and Nardostachys jatamansi fetched the highest price of Rs.300. Some of the medicinal plants species are available seasonally/annually either in community forests $(\mathrm{CF})$, reserve forest (RF), protected forests (PF), khasmal forest (KF), and agroforests (AF) as presented in Table 8.

\section{Discussion}

The forests preserve the biological diversity as well as provide the natural wealth to the human being. But forests are still degrading day by day due to exponential population growth, 
Table 2: Different sources, availability status, and people’s dependency on local resources found in Barsey-Singalila site.

\begin{tabular}{|c|c|c|c|c|c|}
\hline Settlements & Resource types & Source & $\begin{array}{c}\text { Availability } \\
\text { status }\end{array}$ & $\begin{array}{c}\text { Availability } \\
\text { distance }(\mathrm{km})\end{array}$ & $\begin{array}{l}\text { Dependence } \\
\text { index }\left({ }^{*} \mathrm{DI}\right)\end{array}$ \\
\hline Ribdi Bhareng & $\begin{array}{c}\text { Firewood, fodder, wood/poles } \\
\text { (fencing), NTFPs, medicinal } \\
\text { herbs, and litter }\end{array}$ & $\begin{array}{l}\text { Wildlife sanctuary, reserved } \\
\text { forests, khasmal forests, and } \\
\text { private forests }\end{array}$ & Medium & $2-4$ & 0.60 \\
\hline $\begin{array}{l}\text { Gorkhey- } \\
\text { Samanden }\end{array}$ & $\begin{array}{l}\text { Firewood, fodder, wood/poles, } \\
\text { NTFPs and medicinal herbs, and } \\
\text { litter }\end{array}$ & $\begin{array}{l}\text { National park, reserved forests, } \\
\text { and khasmal forests }\end{array}$ & Low & $2-5$ & 0.60 \\
\hline
\end{tabular}

${ }^{*}$ Dependence index (DI) is calculated on a scale of $0-1$.

TABle 3: Amount of collection probability of use (PU) and resource use index (RUI) for important fuelwood species in Barsey-Singalila.

\begin{tabular}{|c|c|c|c|c|c|}
\hline \multirow[b]{2}{*}{ Species (local name) } & \multirow[b]{2}{*}{ Habit } & \multicolumn{4}{|c|}{ Collection (mean) } \\
\hline & & $\mathrm{Kg} \cdot \mathrm{sample}^{-1} \cdot \mathrm{d}^{-1}$ & $\mathrm{Kg} \cdot \mathrm{sample}^{-1} \cdot \mathrm{yr}^{-1}$ & $\begin{array}{c}\text { Probability of use } \\
\text { (PU) }\end{array}$ & $\begin{array}{c}\text { Resource use index } \\
\text { (RUI) }\end{array}$ \\
\hline Alnus nepalensis D.Don (Uttis) & $\mathrm{T}$ & 1.61 & 241.5 & 0.791 & 189.8 \\
\hline Beilschmiedia roxburghiana Nees (Tarsing) & $\mathrm{T}$ & 0.19 & 28.5 & 0.339 & 81.3 \\
\hline Betula cylindrostachys Wall. ex Diels (Saur) & $\mathrm{T}$ & 0.45 & 67.5 & 0.632 & 151.6 \\
\hline $\begin{array}{l}\text { Castanopsis indica (Roxb. ex Lindl.) A.DC. } \\
\text { (Dhalne Katus) }\end{array}$ & $\mathrm{T}$ & 1.44 & 216 & 0.678 & 162.7 \\
\hline $\begin{array}{l}\text { Castanopsis tribuloides (Sm.) A. DC. (Musurey } \\
\text { Katus) }\end{array}$ & $\mathrm{T}$ & 1.33 & 199.5 & 0.7 & 168 \\
\hline Chukrasia tabularis A.Juss. (Bogipoma) & $\mathrm{T}$ & 1.21 & 181.5 & 0.271 & 65.0 \\
\hline $\begin{array}{l}\text { Cryptomeria japonica (Thunb. ex L.f.) D.Don } \\
\text { (Dhuppi) }\end{array}$ & $\mathrm{T}$ & 0.25 & 37.5 & 0.293 & 70.3 \\
\hline $\begin{array}{l}\text { Dendrocalamus hamiltonii Nees \& Arn. ex } \\
\text { Munro (Bans) }\end{array}$ & $\mathrm{T}$ & 1.23 & 184.5 & 0.745 & 178.8 \\
\hline Edgeworthia gardneri (Wall.) Meisn. (Argeli) & S & 0.57 & 85.5 & 0.542 & 130.0 \\
\hline Engelhardtia spicata Lechen ex Blume (Mauwa) & $\mathrm{T}$ & 0.45 & 67.5 & 0.587 & 140.8 \\
\hline Eurya acuminata DC. (Zhingane) & $\mathrm{T}$ & 0.85 & 127.5 & 0.519 & 124.5 \\
\hline Leucosceptrum canum Sm. (Ghurpis) & $\mathrm{T}$ & 0.62 & 93 & 0.565 & 135.6 \\
\hline Lithocarpus pachyphyllus (Kurz) Rehder & $\mathrm{T}$ & 0.12 & 18 & 0.18 & 43.2 \\
\hline Lyonia ovalifolia (Wall.) Drude (Angaree) & $\mathrm{T}$ & 0.43 & 64.5 & 0.203 & 48.7 \\
\hline Macaranga indica Wight (Malata) & $\mathrm{T}$ & 0.66 & 99 & 0.361 & 86.6 \\
\hline $\begin{array}{l}\text { Macaranga denticulata (Blume) Müll. Arg. } \\
\text { (Sanomalata) }\end{array}$ & $\mathrm{T}$ & 0.13 & 19.5 & 0.384 & 92.1 \\
\hline Machilus edulis King ex Hook.f. (Rani Kawlo) & $\mathrm{T}$ & 0.31 & 46.5 & 0.406 & 97.4 \\
\hline Maesa chisia Buch.-Ham. ex D. Don (Bilaune) & $\mathrm{T}$ & 0.24 & 36 & 0.406 & 97.4 \\
\hline $\begin{array}{l}\text { Nyssa javanica (Blume) Wangerin } \\
\text { (Lekhchilaune) }\end{array}$ & $\mathrm{T}$ & 0.14 & 21 & 0.293 & 70.3 \\
\hline $\begin{array}{l}\text { Prunus cerasoides Buch.-Ham. ex D.Don } \\
\text { (Paiyun) }\end{array}$ & $\mathrm{T}$ & 0.54 & 81 & 0.497 & 119.2 \\
\hline $\begin{array}{l}\text { Lithocarpus fenestratus (Roxb.) Rehder } \\
\text { (Arkahulo) }\end{array}$ & $\mathrm{T}$ & 0.59 & 88.5 & 0.18 & 43.2 \\
\hline Quercus lamellosa Sm. (Bajranth) & $\mathrm{T}$ & 1.62 & 243 & 0.158 & 37.9 \\
\hline Quercus lineata Blume (Phlant) & $\mathrm{T}$ & 0.98 & 147 & 0.135 & 32.4 \\
\hline Rhododendron arboretum Sm. (Laligurans) & $\mathrm{T}$ & 0.45 & 67.5 & 0.09 & 21.6 \\
\hline Schima wallichii Choisy (Chilaune) & $\mathrm{T}$ & 0.86 & 129 & 0.429 & 102.9 \\
\hline Symplocos theifolia (Kharane) & $\mathrm{T}$ & 0.23 & 34.5 & 0.316 & 75.8 \\
\hline $\begin{array}{l}\text { Symplocos sumuntia Buch.-Ham. ex D.Don } \\
\text { (Kholme) }\end{array}$ & $\mathrm{T}$ & 0.12 & 18 & 0.339 & 81.3 \\
\hline Tsuga dumosa (D.Don) Eichler (Thingre salla) & $\mathrm{T}$ & 0.11 & 16.5 & 0.113 & 27.1 \\
\hline Viburnum nervosum D.Don (Ashare) & $\mathrm{T}$ & 0.67 & 100.5 & 0.226 & 54.2 \\
\hline Meliosma arnottiana (Wight) Walp (Dabdabe) & $\mathrm{T}$ & 0.13 & 19.5 & 0.18 & 43.2 \\
\hline (Aankhle) & $\mathrm{T}$ & 0.11 & 16.5 & 0.135 & 32.4 \\
\hline Arundo donax (Narkat) & S & 1.68 & 252 & 0.813 & 195.1 \\
\hline
\end{tabular}

$\mathrm{T}$, tree; $\mathrm{S}$, shrub. 
TABLE 4: Traditional resource use pattern in the Barsey-Singalila site.

\begin{tabular}{|c|c|c|c|c|c|}
\hline \multirow{2}{*}{ Resource } & \multirow{2}{*}{ Extraction process } & \multirow{2}{*}{ Species under use } & \multirow{2}{*}{ Frequency } & \multicolumn{2}{|c|}{$\begin{array}{c}\text { Average } \\
\text { consumption }\end{array}$} \\
\hline & & & & $\begin{array}{c}\mathrm{kg} / \\
\mathrm{hh} / \mathrm{d}\end{array}$ & $\begin{array}{c}\mathrm{kg} / \mathrm{hh} / \\
\mathrm{yr}\end{array}$ \\
\hline \multicolumn{6}{|l|}{ Ribdi Bhareng } \\
\hline Fuelwood & $\begin{array}{l}\text { Felling, lopping, and } \\
\text { collecting }\end{array}$ & & $\begin{array}{c}\text { Morning 2-3 times } \\
\text { a day }\end{array}$ & 20.3 & 7409.5 \\
\hline Fodder & $\begin{array}{l}\text { Felling, chopping, and } \\
\text { collecting }\end{array}$ & & $\begin{array}{c}\text { Morning 2-3 times } \\
\text { a day }\end{array}$ & 18 & 6570 \\
\hline${ }^{*}$ Timber & Felling and sawing & $\begin{array}{c}\text { Eragrostis gangetica (Bansho/Ghini), Persicaria } \\
\text { chinensis (Ratnaulo), Persicaria chinensis (Ratnaulo), } \\
\text { and Strobilanthus sp. (Ankhley) }\end{array}$ & $\begin{array}{l}2-3 \text { times in } \\
\text { between } 5 \text { and } 10 \\
\text { years }\end{array}$ & - & $7.5 \mathrm{~cm}^{3}$ \\
\hline $\begin{array}{l}\text { NTFPs and } \\
\text { medicinal } \\
\text { plants }\end{array}$ & $\begin{array}{l}\text { Felling, chopping, umbel } \\
\text { picking, uprooting, and } \\
\text { collecting }\end{array}$ & & $\begin{array}{l}\text { Morning 2-3 times } \\
\text { a week }\end{array}$ & 0.4 & 146 \\
\hline Litter & Collecting & & $>2-3$ times a day & 12 & 4380 \\
\hline \multicolumn{6}{|c|}{ Gorkhey-Samanden } \\
\hline Fuelwood & $\begin{array}{l}\text { Felling, lopping, and } \\
\text { collecting }\end{array}$ & & $\begin{array}{c}\text { Morning 2-3 times } \\
\text { a day }\end{array}$ & 20 & 7300 \\
\hline Fodder & $\begin{array}{l}\text { Felling, chopping, and } \\
\text { collecting }\end{array}$ & & $\begin{array}{c}\text { Morning 2-3 times } \\
\text { a day }\end{array}$ & 15 & 5475 \\
\hline Timber & Felling and sawing & $\begin{array}{c}\text { Eragrostis gangetica (Bansho/Ghini), Persicaria } \\
\text { chinensis (Ratnaulo), Persicaria chinensis (Ratnaulo), } \\
\text { and Strobilanthus sp. (Ankhley) }\end{array}$ & $\begin{array}{l}2-3 \text { times in } \\
\text { between } 5-10 \\
\text { years }\end{array}$ & - & $6 \mathrm{~cm}^{3}$ \\
\hline $\begin{array}{l}\text { NTFPs and } \\
\text { medicinal } \\
\text { plants }\end{array}$ & $\begin{array}{l}\text { Felling, chopping, umbel } \\
\text { picking, uprooting, and } \\
\text { collecting }\end{array}$ & & $\begin{array}{l}\text { Morning 2-3 times } \\
\text { a week }\end{array}$ & 0.5 & 182.5 \\
\hline Litter & Collecting & & $>2-3$ times a day & 10 & 3650 \\
\hline
\end{tabular}

${ }^{*}$ Timber is required in between 10 and 15 years for house construction.

TABLE 5: Annual resource production and actual demand in study villages under Barsey-Singalila range.

\begin{tabular}{|c|c|c|c|c|c|c|c|c|}
\hline \multirow[b]{2}{*}{ Resource } & \multicolumn{4}{|c|}{ Ribdi Bhareng } & \multicolumn{4}{|c|}{ Gorkhey-Samanden } \\
\hline & $\begin{array}{c}\text { Demand } \\
\left.\text { (ton } / \mathrm{cm}^{3} / \mathrm{yr}\right)\end{array}$ & Production (ton/yr) & S & $\mathrm{PE}$ & Demand (ton $/ \mathrm{cm}^{3} / \mathrm{yr}$ ) & Production (ton/yr) & S & $\mathrm{PE}$ \\
\hline Fuelwood & 2393.27 & 3.23 & 2390.04 & 1434.02 & 445.3 & 0.12 & 445.18 & 267.11 \\
\hline Fodder & 2122.11 & 46.51 & 2075.6 & 1245.36 & 333.98 & 3.66 & 330.32 & 198.19 \\
\hline Timber & 2422.5 & 64.6 & 2357.9 & 1414.74 & 366 & 6.1 & 359.9 & 215.94 \\
\hline NTFPs & 47.16 & 29.47 & 17.69 & 10.61 & 11.13 & 5.7 & 5.43 & 3.26 \\
\hline Litter & 1414.74 & 2.26 & 1412.48 & 847.49 & 222.65 & 0.43 & 222.22 & 133.33 \\
\hline Food crops* & 279.07 & 69.77 & 209.3 & 125.58 & 52.70 & 13.18 & 39.52 & 23.71 \\
\hline
\end{tabular}

DI, dependency index from Table 1. Shortfall $(S)=D-P$. Possible extraction $(\mathrm{PE})=S \times$ DI. * Purchasing of food crops/items.

poverty, and lack of awareness about the ecosystem services [8]. More than 350 million people were living inside or on the fringes of forests across the world, out of which 60 million were largely dependent for sustenance [6]. Forests in most developing countries are under anthropogenic pressure from resource use by marginalized communities for subsistence [7, 20, 21]. The condition is considered more perilous in Asia and Africa, where loss of forests during a ten-year period in the later part of the $20^{\text {th }}$ century was estimated to 163 million ha, of which 154 million ha (94.5\%) was in the tropics alone [22].

Resource use data suggests that resource need is collected from a certain distance in the forest area which will be far from the houses. The dependency index of study sites was found much higher than the dependency rate of households as estimated in the midhills of eastern Nepal (40\%) [23]. The households with low income and small fragmented land are more dependent on the forest resources [24]. Type and degree of dependence varies from place to place. It is highest among the families living within forest areas. Such families (about 10\% of the households) have no or very small landholdings. Others who significantly depend for their household and day to day requirements as well as for certain specific (such as medicinal) uses on neighbouring forests (about 50\%) include not only those principally associated with agriculture or allied activities but also involved with forests one way or the other [25].

Whereas, the average fuelwood consumption in the area is comparatively higher than the fuelwood consumption 
TABLe 6: Preference-wise ranking of fodder species in study sites.

\begin{tabular}{|c|c|c|}
\hline Most preferred (local name) & Preferred (local name) & Least preferred (local name) \\
\hline $\begin{array}{l}\text { Ribdi Bhareng } \\
\text { Arundo donax L. (Narkat) } \\
\text { Lithocarpus pachyphyllus (Kurz) Rehder } \\
\text { (Bantey) } \\
\text { Litsea elongata (Nees) Hook (Pahenley) } \\
\text { Sida acuta Burm. (Balu) } \\
\text { Acer calcaratum Gagnep. (Kapshee) } \\
\text { Meliosma arnottiana (Wight) Walp } \\
\text { (Dabdabe) } \\
\text { Litsea sp. (Timmur) }\end{array}$ & $\begin{array}{c}\text { Pennisetum purpureum Schumach. (Napier) } \\
\text { Eragrostis gangetica (Roxb.) Steud. (Bansho/Ghini) } \\
\text { Persicaria chinensis (L.) H. Gross (Ratnaulo) } \\
\text { Machilus sp. (Kawlo) } \\
\text { Arundo donax L. (Narkat) } \\
\text { Strobilanthus sp. (Ankhley) } \\
\text { Quercus lineata Blume (Phlant) } \\
\text { Evodia fraxinifolia (Hook.) Benth. (Khanakpa) } \\
\text { Yushania maling (Gamble) R.B. Majumdar \& } \\
\text { Karthik.(Malingo) } \\
\text { Amiley ghans } \\
\text { Toona ciliata M. Roem. (Tuni) } \\
\text { Actinidia strigosa Hook. F. \& Thomson ex Benth } \\
\text { (Thekiphal) }\end{array}$ & $\begin{array}{c}\text { Sida acuta Burm. (Balu) } \\
\text { Litsea sp. (Timmur) } \\
\text { Rubus sp. (Berry) } \\
\text { Ficus elastica Roxb. (Lishey) } \\
\text { Zea mays L. (Maize residue) } \\
\text { Eragrostis gangetica (Roxb.) Steud. } \\
\text { (Bansho/Ghini) }\end{array}$ \\
\hline $\begin{array}{l}\text { Gorkhey-Samanden } \\
\text { Arundo donax L. (Narkat) } \\
\text { Lithocarpus pachyphyllus (Kurz) Rehder } \\
\text { (Bantey) } \\
\text { Litsea elongata (Nees) Hook (Pahenley) } \\
\text { Sida acuta Burm. (Balu) } \\
\text { Acer calcaratum Gagnep. (Kapshee) } \\
\text { Meliosma arnottiana (Wight) Walp. } \\
\text { (Dabdabe) } \\
\text { Litsea sp. (Timmur) }\end{array}$ & $\begin{array}{c}\text { Pennisetum purpureum Schumach. (Napier) } \\
\text { Poa sp. (Bansho/Ghini) } \\
\text { Persicaria chinensis (L.) H. Gross (Ratnaulo) } \\
\text { Machilus sp. (Kawlo) } \\
\text { Arundo donax L. (Narkat) } \\
\text { Strobilanthus sp. (Ankhley) } \\
\text { Quercus lineata Blume (Phlant) } \\
\text { Evodia fraxinifolia (Hook.) Benth. } \\
\text { Yushania maling (Gamble) R.B. Majumdar \& } \\
\text { Karthik.(Malingo) } \\
\text { Amiley ghans } \\
\text { Toona ciliata M. Roem.(Tuni) } \\
\text { Actinidia strigosa Hook. F. \& Thomson ex Benth } \\
\text { (Thekiphal) }\end{array}$ & $\begin{array}{c}\text { Sida acuta Burm. (Balu) } \\
\text { Litsea sp. (Timmur) } \\
\text { Rubus sp. (Berry) } \\
\text { Ficus elastica Roxb. (Lishey) } \\
\text { Zea mays L. (Maize residue) } \\
\text { Eragrostis gangetica (Roxb.) Steud. } \\
\text { (Bansho/Ghini) }\end{array}$ \\
\hline
\end{tabular}

1, abundant; 2, common; 3 , rare.

amount as reported for Garhwal Himalaya [26] but lesser than the amount measured in Kedarnath region [27]. So, it honestly proves that pressure on resources is considerably high. Yearly production of the resource was also comparatively much lesser than the requirement for households of both the study sites, and this result can be compared with a study held in Niti valley where fuelwood consumption was much lower than the consumption amount of other greater Himalayan villages [28]. The average fuelwood consumption data is also compared with data in Kumaon villages, where per capita consumption amount was estimated to $3.14 \mathrm{~kg} /$ day [29]. Since fuelwood collection forms the highest and the most frequent use of forests followed by timber and fodder collection for livestock [25]. Although Arundo donax shows high RUI, it is available at farmland. Alnus nepalensis, Dendrocalamushamiltoni, and Castanopsis tribuloides having high RUI are available at the forest including Alnus nepalensis depicting high pressure on the species. Definitely, these species having high PU show preferences in the villages. Thus, a comparatively larger village with more population will aggravate utilization pressures.
Resource production is far lesser than the demand as evident from the present survey. The area is characterized by sparse vegetation cover, low primary productivity, and short growing season and is thus highly susceptible to irreversible changes of natural habitats $[28,30]$. A huge shortfall in resource availability predicts the possible extraction of resources from the surrounding forests (namely, the khasmal forests, protected areas, and agroforestry areas). And the whole can also be compared with the total available fodder in Central Himalayan village [31].

There is a huge gap between timber demand and production which is required for construction of house and goths/cowshed, and such case is evident from timber demand at Ribdi-Bhareng and wood production rate, thereby, leading to huge shortfall. Considering the fact that a very small fraction of firewood comes from the agriculture fields, various resources demand and production in the study villages are attributed to production and utilization patterns. 
TABLe 7: NTFPs/wild edibles (1 US\$=Rs. 72 approx.).

\begin{tabular}{|c|c|c|c|c|c|}
\hline Species (local name) & Usage & $\begin{array}{l}\text { Degree of } \\
\text { use }\end{array}$ & $\begin{array}{l}\text { Availability } \\
\text { status }\end{array}$ & $\begin{array}{c}\text { People's } \\
\text { dependency }\end{array}$ & $\begin{array}{l}\text { Market price } \\
\left(\mathrm{Rs} \cdot \mathrm{kg}^{-1}\right)\end{array}$ \\
\hline Polygonum molle D. Don (Thotnee) & $\begin{array}{l}\text { Fodder, NTFP, and } \\
\text { edible }\end{array}$ & Low & Abundant & High & - \\
\hline Artemisia vulgaris L. (Titeypati) & NTFP and medicine & High & Abundant & High & - \\
\hline Zanthoxylum sp. (Eirmong) & NTFP and medicine & Low & Abundant & Low & 50.0 \\
\hline $\begin{array}{l}\text { Himalayacalamus hookerianus (Munro) } \\
\text { Stapleton (Paraeing) }\end{array}$ & $\begin{array}{l}\text { Roofing, fencing, } \\
\text { and NTFP }\end{array}$ & High & Moderate & High & 50.0 \\
\hline $\begin{array}{l}\text { Yushania maling (Gamble) R. B. Majumdar \& } \\
\text { Karthik.(Malingo) }\end{array}$ & Roofing and NTFP & Medium & Rare & High & 80.0 \\
\hline $\begin{array}{l}\text { Astilbe rivularis Buch.-Ham. ex D.Don } \\
\text { (Budookhati) }\end{array}$ & Medicine & Low & Rare & Medium & 30.0 \\
\hline Diplazium sp.(Ninguro) & NTFP and edible & Medium & Abundant & High & 10.0 \\
\hline Evodia fraxinifolia (Hook.) Benth. (Khanakpa) & NTFP and remedial & Low & Rare & High & - \\
\hline Heracleum wallichii DC (Chimphing) & NTFP and medicine & Low & Rare & High & 30.0 \\
\hline Litsea cubeba (Lour.) Pers. (Siltimur) & NTFP and medicine & Medium & Abundant & High & 54.0 \\
\hline $\begin{array}{l}\text { Pentapanax castanopsidicola Hayata } \\
\text { (Chinday) }\end{array}$ & NTFP and edible & Medium & Abundant & Medium & 30.0 \\
\hline Rhododendron arboreum Sm. (Gurans) & NTFP and medicine & Medium & Abundant & High & - \\
\hline Rubus ellipticus Sm. (Aiselu) & NTFP and edible & Low & Abundant & Medium & - \\
\hline Urtica dioica L. (Sisnu) & $\begin{array}{l}\text { NTFP, fibre, and } \\
\text { edible }\end{array}$ & High & Abundant & High & 25.0 \\
\hline $\begin{array}{l}\text { Zanthoxylum acanthopodium DC. (Bokey } \\
\text { timur) }\end{array}$ & $\begin{array}{l}\text { NTFP, edible, and } \\
\text { medicine }\end{array}$ & Low & Rare & Medium & 20.0 \\
\hline $\begin{array}{l}\text { Actinidia strigosa Hook. F. \& Thomson ex } \\
\text { Benth (Thekiphal) }\end{array}$ & NTFP and edible & Medium & Rare & Medium & 20.0 \\
\hline $\begin{array}{l}\text { Pyrus pashia Buch.-Ham. ex D.Don (Jangali } \\
\text { Mel) }\end{array}$ & $\begin{array}{l}\text { NTFP, edible, and } \\
\text { medicine }\end{array}$ & Medium & Rare & Medium & 20.0 \\
\hline Agaricus sp. (Local) & NTFP and edible & High & Rare & High & 150.0 \\
\hline Arisema sp. (Gurbo) & NTFP and edible & Low & Rare & Medium & 30.0 \\
\hline $\begin{array}{l}\text { Castanopsis indica (Roxb. ex Lindl.) A.DC. } \\
\text { (Dhalne Katus) }\end{array}$ & NTFP and edible & Low & Rare & Medium & 20.0 \\
\hline
\end{tabular}

The higher price is attributed being the species grow in high altitudes and in rare status [32]. Similarly, mushroom considered as valuable wild edible fetch higher market cost. This income can be compared with the valueadded products of most selected wild edibles which derived maximum economic returns in Garhwal Himalaya for Hippophae salicifolia followed by Rhododendron arboreum and Spondias pinnata and minimum for Myrica esculenta [33]. Communities of both the villages use local medicinal plants for treatment of health problems related to indigestion, antispasmodic, chronic fever, dysentery, and influenza, for which different parts (e.g., root, fruit, leaf, and sometimes entire plant) are used. These medicinal plants are found to be distributed within an elevation range between 800 and $3600 \mathrm{~m}$ asl in the Himalaya [34]. Preferred species are collected regardless of their availability for fuelwood and other uses, which is causing high pressure on a small group of plants. Marketing of medicinal herbs is inefficient, informal, secretive, and opportunistic. As a result, the raw material supply situation is shaky, unsustainable, and exploitative, which in turn leads to depletion of the resource base exploiting of the rural people (who are the real stewards of the resource), adulteration, and nonavailability of quality herbal drugs for domestic consumption as well as for exports $[35,36]$. In a conclusion, local ecosystem suffers from shortage of suitable resource management policies in one; similarly, delay in implementation of associated policies may also result in gradual loss of biodiversity in another. The regional initiatives taken by G. B. Pant National Institute of Himalayan Environment (NIHE) under Khangchendzonga Landscape Conservation and Development Initiative- (KLCDI-) India programme have been widely appreciated. KLCDI is one of such transboundary programme where partner countries may work together taking the above approaches.

\section{Conclusion}

Transboundary areas are more vulnerable in terms of encroachment such as tourist trekking, carrying of business items, animal grazing, and collection of medicinal/wild plants, as evidenced in the Barsey-Singalila transboundary area. Sustainable use of the resource in the area is much required so that livelihood of the local people be improved. Production using local and productive fodder species should be emphasized. Fuel, fodder, and wild edibles including medicinal herbal are diverse, but there may a great threat to those species of protected areas. Our results emphasized the need for regular assessment of forest resources, particularly in protected areas. The afforestation of degraded, uncultivated, and marginal lands through high-quality fuel species 
TABLE 8: Medicinal herbs from the study sites.

\begin{tabular}{|c|c|c|c|c|c|c|c|c|}
\hline $\begin{array}{l}\text { Species } \\
\text { (local name) }\end{array}$ & $\begin{array}{c}\text { Elevation } \\
(\mathrm{m})\end{array}$ & Habit & $\begin{array}{c}\text { Availability } \\
\text { time }\end{array}$ & Parts use & $\begin{array}{c}\text { Colour/ } \\
\text { Taste }\end{array}$ & Usage & $\begin{array}{l}\text { Consumption } \\
\left(\mathrm{kg} \mathrm{hh}^{-1} \cdot \mathrm{yr}^{-1}\right)\end{array}$ & Source \\
\hline Acorus calamus L. (Bonjho) & $800-2500$ & Herb & Perennial & Root & $\begin{array}{l}\text { White/ } \\
\text { bitter }\end{array}$ & Bone fracture & 5 & $\mathrm{CF}$ \\
\hline Artemisia vulgaris L. (Titeypati) & $800-2000$ & Herb & Perennial & $\begin{array}{c}\text { Root and } \\
\text { leaves }\end{array}$ & $\begin{array}{l}\text { Green/ } \\
\text { bitter }\end{array}$ & $\begin{array}{l}\text { Antiseptic and } \\
\text { asthma }\end{array}$ & 400 & $\mathrm{~F} / \mathrm{RF}$ \\
\hline $\begin{array}{l}\text { Astilbe rivularis Buch.-Ham. ex } \\
\text { D.Don (Budookhat) }\end{array}$ & $1600-3300$ & Shrub & Perennial & Root & $\begin{array}{l}\text { Green/ } \\
\text { bitter }\end{array}$ & Dysentery & 200 & $\mathrm{RF}$ \\
\hline $\begin{array}{l}\text { Eupatorium cannabinum } \\
\text { L. (Banmara) }\end{array}$ & $800-2500$ & Herb & Perennial & Leaf & $\begin{array}{l}\text { Green/ } \\
\text { bitter }\end{array}$ & Bleeding & - & $\mathrm{AF} / \mathrm{CF}$ \\
\hline $\begin{array}{l}\text { Evodia fraxinifolia (Hook.) } \\
\text { Benth. (Khanakpa) }\end{array}$ & $1500-2500$ & Tree & August & Fruit & $\begin{array}{l}\text { Green/ } \\
\text { bitter }\end{array}$ & Indigestion & 500 & $\mathrm{RF}$ \\
\hline $\begin{array}{l}\text { Heracleum wallichii DC. } \\
\text { (Chimphing) }\end{array}$ & $1500-2500$ & Herb & September & Fruit & Red/bitter & Influenza & 600 & $\mathrm{KF}$ \\
\hline $\begin{array}{l}\text { Centella asiatica (L.) Urb. } \\
\text { (Dalleypat) }\end{array}$ & $1000-2500$ & Herb & Perennial & Leaf & Sour & Throat pain & - & $\begin{array}{c}\text { Farm/ } \\
\mathrm{AF}\end{array}$ \\
\hline $\begin{array}{l}\text { Nardostachys jatamansi (D.Don) } \\
\text { DC. (Jatamansi) }\end{array}$ & $3600-4800$ & Herb & Perennial & Root & $\begin{array}{c}\text { Green/ } \\
\text { bitter }\end{array}$ & Antispasmodic & 100 & $\mathrm{CF} / \mathrm{AF}$ \\
\hline $\begin{array}{l}\text { Swertia chirata Buch.-Ham. ex } \\
\text { Wall (Chirawto) }\end{array}$ & $1600-2600$ & Herb & July & $\begin{array}{l}\text { Whole } \\
\text { plant }\end{array}$ & $\begin{array}{l}\text { Green/ } \\
\text { bitter }\end{array}$ & Chronic fever & 1000 & $\mathrm{PF}$ \\
\hline $\begin{array}{l}\text { Amomum subulatum Roxb. } \\
\text { (Elaichi) }\end{array}$ & $500-1900$ & Herb & October & Fruit & $\begin{array}{l}\text { Grey/ } \\
\text { sweet }\end{array}$ & Spice & 500 & $\mathrm{KF} / \mathrm{AF}$ \\
\hline Elaeocarpus sp. (Rudraksha) & $1000-1800$ & Tree & November & Fruit & $\begin{array}{l}\text { Green/ } \\
\text { sour }\end{array}$ & Stomachache & 600 & $\mathrm{KF} / \mathrm{AF}$ \\
\hline Entada sp. & $1500-2500$ & Tree & Perennial & Flower & Alkaloid & Indigestion & 50 & $\mathrm{KF} / \mathrm{AF}$ \\
\hline $\begin{array}{l}\text { Cinnamomum tamala (Buch.- } \\
\text { Ham.) T.Nees \& Eberm. (Tejpat) }\end{array}$ & $800-2500$ & Tree & Perennial & Leaf & $\begin{array}{l}\text { Green/ } \\
\text { tasteless }\end{array}$ & Spices & 100 & $\mathrm{CF} / \mathrm{AF}$ \\
\hline $\begin{array}{l}\text { Thysanolaena latifolia (Roxb. ex } \\
\text { Hornem.) Honda }\end{array}$ & $300-4800$ & Shrub & August & Root & $\begin{array}{l}\text { White/ } \\
\text { tasteless }\end{array}$ & Diarrhea & 1000 & $\mathrm{PF} / \mathrm{AF}$ \\
\hline Zanthoxylum sp. (Timbur) & $1600-2600$ & Tree & November & Fruit & Red/hot & Antispasmodic & 500 & $\mathrm{RF} / \mathrm{KF}$ \\
\hline Phyllanthus emblica L. (Amla) & $500-1500$ & Tree & Perennial & Fruit & $\begin{array}{l}\text { Green/ } \\
\text { sour }\end{array}$ & $\begin{array}{l}\text { Stomach } \\
\text { problems }\end{array}$ & 600 & $\mathrm{KF} / \mathrm{AF}$ \\
\hline $\begin{array}{l}\text { Rumex nepalensis Spreng. } \\
\text { (Halhale) }\end{array}$ & $800-1800$ & Herb & Perennial & Root & Tasteless & Dysentery & - & $\begin{array}{l}\text { Farm/ } \\
\mathrm{AF}\end{array}$ \\
\hline Rhus succedanea L. (Arkhol) & $800-2500$ & Tree & November & Fruit & Grey/sour & Dysentery & 100 & $\mathrm{CF} / \mathrm{AF}$ \\
\hline
\end{tabular}

RF, reserved forest; PF, protected forest; $F$, farm.

in the villages might reduce pressure. Energy value of these species also needs to be determined.

The study further suggests that providing alternate and nonconventional energy sources such as solar cookers and fuel efficient portable ovens to the inhabitants at subsidized rates could reduce the pressure on nearby forests. However, the local government has supported the community people through supplying LPG stoves and cylinders at free of cost which may not be in sufficient quantity. Besides, judicious harvest of resources considering the production rate is ever appreciated. Similarly, government policies involving resource management can impact development processes. Further alternative energy sources are to be generated such as the use of biobriquettes (matula) as fuelwood.

\section{Data Availability}

The data used to support the findings of this study are available from the corresponding author upon request.

\section{Conflicts of Interest}

The authors declare that they have no conflicts of interest.

\section{Acknowledgments}

The authors are grateful to the Director, G. B. Pant National Institute for Himalayan Environment, for providing necessary support and facilities for carrying out research. Financial support and cooperation received from International Centre for Integrated Mountain Development (ICIMOD) during KLCDI-India programme implementation are duly acknowledged. The kind of support extended by The Mountain Institute India is acknowledged. The authors are also grateful to Forest, Environment, and Wildlife Management Department, Government of Sikkim, and Directorate of Forests, West Bengal, for affording support during our field works. Last, the support and cooperation extended by the Panchayat members and community people of Ribdi-Bhareng and Gorkhey-Samanden are deeply appreciated. 


\section{References}

[1] D. K. Langat, E. K. Maranga, A. A. Aboud, and J. K. Cheboiwo, "Role of forest resources to local livelihoods: the case of East Mau forest ecosystem, Kenya," International Journal of Forestry Research, vol. 2016, Article ID 4537354, 10 pages, 2016.

[2] S. Chao, Forest People: Numbers across the World, Forest Peoples Program, Moreton-in-Marsh, UK, 2012.

[3] P. Vedeld, A. Angelsen, J. Bojö, E. Sjaastad, and G. Kobugabe Berg, "Forest environmental incomes and the rural poor," Forest Policy and Economics, vol. 9, no. 7, pp. 869-879, 2007.

[4] C. Shackleton and S. Shackleton, "The importance of non timber forest products in rural livelihood security and as safety nets: a review of evidence from South Africa," South African Journal of Science, vol. 100, no. 11-12, pp. 658-664, 2004.

[5] C. M. Shackleton and S. E. Shackleton, "Household wealth status and natural resource use in the Kat River valley, South Africa," Ecological Economics, vol. 57, no. 2, pp. 306-317, 2006.

[6] World Bank, Agriculture and Rural Development Sector Unit, and South Asia Region, India: Unlocking Opportunities for Forest Dependent People, The World Bank, Washington, DC, USA, 2006.

[7] MoEF Ministry of Environment and Forest, Government of India, State of Environment Report, Vol. 126, Ministry of Environment and Forest, New Delhi, India, 2009.

[8] K. A. Khan, A. Musavi, J. A. Khan, and K. Ahmad, "Livelihood of two local communities and their dependence on forest resources: a case study from Western Himalaya, Dachigam national park, Jammu \& Kashmir, India," Journal of Human Resource and Sustainability Studies, vol. 6, no. 4, pp. 294-305, 2018.

[9] N. Chettri, E. Sharma, D. C. Deb, and R. C. Sundriyal, "Impact of firewood extraction on tree structure, regeneration and woody biomass productivity in a trekking corridor of the Sikkim Himalaya," Mountain Research and Development, vol. 22, no. 2, pp. 150-158, 2002.

[10] M. Arnold, G. Kohlin, R. Persson, and G. Shepherd, Fuelwood Revisited: What Has Changed in the Last Decade?, Centre for International Forestry Research, Bogor, Indonesia, 2003.

[11] D. Dhyani, R. K. Maikhuri, K. S. Rao et al., "Basic nutritional attributes of Hippophae salicifolia (Seabuckthorn) populations from Uttarakhand Himalaya, India," Current Science, vol. 92, no. 8, pp. 1148-1152, 2007.

[12] R. K. Maikhuri, L. S. Rawat, V. Negi, and V. K. Purohit, EcoFriendly Appropriate Technologies for Sustainable Development of Rural Ecosystems in Central Himalaya, G. B. Pant Institute of Himalayan Environment and Development, Almora, Uttarakhand, 2007.

[13] R. K. Maikhuri, V. Negi, L. S. Rawat, and V. K. Purohit, Promoting Value Addition in Potential Wild Edibles of Central Himalaya for Sustainable Livelihood and Small Scale Enterprise Development, G. B. Pant Institute of Himalayan Environment and Development, Almora, Uttarakhand, 2007.

[14] M. Singh, A. Chettri, A. Pandey, S. Sinha, K. K. Singh, and H. K. Badola, "In vitro propagation and phytochemical assessment of Aconitum ferox wall: a threatened medicinal plant of Sikkim Himalaya," Proceedings of the National Academy of Sciences, India Section B: Biological Science, vol. 90, pp. 1-9, 2019.
[15] P. Kandel, N. Chettri, R. P. Chaudhary et al., "Plant diversity of the Kangchenjunga Landscape, Eastern Himalayas," Plant Diversity, vol. 41, no. 3, pp. 153-165, 2019.

[16] M. I. Clarke, "Activity modelling a research tool or a practical planning technique?” in Behavioral Research for Transport PolicyVol. 123, VNU Science Press, Utrecht, Netherlands, 1986.

[17] S. S. Samant, U. Dhar, and R. S. Rawal, “Assessment of fuel resource diversity and utilization patterns in Askot wildlife sanctuary in Kumaun Himalaya, India, for conservation and management," Environmental Conservation, vol. 27, no. 1, pp. 5-13, 2000.

[18] M. S. Rana, B. Rana Sakshi, B. Sakshi, and S. S. Samant, "Extraction, utilization pattern and prioritization of fuel resources for conservation in Manali wildlife sanctuary, Northwestern Himalaya," Journal Mountain Science, vol. 9, pp. 580-588, 2012.

[19] D. Mueller-Dombois and H. Ellenberg, Aims and Methods of Vegetation Ecology, John Willey and Sons, New York, NY, USA, 1974.

[20] P. Davidar, S. Sahoo, P. C. Mammen et al., "Assessing the extent and causes of forest degradation in India: where do we stand?" Biological Conservation, vol. 143, no. 12, pp. 2937-2944, 2010.

[21] FSI, Forest Survey of India, India State of Forest Report, Forest Survey of India (Ministry of Environment and Forests), New Delhi, India, 2011.

[22] FAO Food and Agriculture Organization, Non-Wood Forest Products in Nutrition, FAO/GOI Expert Consultation on Non Wood Forest Products, Yogyakarta, Indonesia, 1995.

[23] D. Pant, S. Thapa, A. Singh, M. Bhattarai, and D. Molden, Integrated Management of Water, Forest and Land Resources in Nepal: Opportunities for Improved Livelihood, International Water Management Institute, Colombo, Sri Lanka, 2005.

[24] A. J. Dougill, J. G. Soussan, E. Kiff et al., "Impacts of community forestry on farming system sustainability in the middle hills of Nepal," Land Degradation \& Development, vol. 12, no. 3, pp. 261-276, 2001.

[25] M. Junaid Jazib, "Forest dependence and the utilization patterns of locals in the PirPanjal Himalayas," Civil and Enviromental Research, vol. 7, no. 1, pp. 78-83, 2015.

[26] A. Awasthi, S. K. Uniyal, G. S. Rawat, and A. Rajvanshi, "Forest resource availability and its use by the migratory villages of Uttarkashi, Garhwal Himalaya (India)," Forest Ecology and Management, vol. 174, no. 1-3, pp. 13-24, 2003.

[27] G. Singh, G. S. Rawat, and D. Verma, "Comparative study of fuelwood consumption by villagers and seasonal "Dhaba owners" in the tourist affected regions of Garhwal Himalaya, India," Energy Policy, vol. 38, no. 4, pp. 1895-1899, 2010.

[28] M. Mitra, A. Kumar, B. S. Adhikari, and G. S. Rawat, "Fuelwood resources and their use pattern by Bhotia community in Niti Valley, Western Himalaya," Botanica Orientalis: Journal of Plant Science, vol. 11, pp. 1-6, 2017.

[29] V.S. Negi and R. K. Maikhuri, "Forest resources consumption pattern in Govind wildlife sanctuary, Western Himalaya, India," Journal of Environmental Planning and Management, vol. 60, no. 7, pp. 1235-1252, 2017.

[30] B. C. Joshi, R. S. Rawal, K. Chandra Sekar, and A. Pandey, "Quantitative ethnobotanical assessment of woody species in a representative watershed of West Himalaya, India," Energy, Ecology and Environment, vol. 4, no. 2, pp. 56-64, 2019.

[31] S. Narayan and R. C. Sundriyal, "Fuelwood and fodder use and deficit pattern in Central Himalayan village," Nature and Science, vol. 7, no. 4, pp. 85-88, 2009. 
[32] H. Meilby, C. Smith-Hall, A. Byg et al., "Are forest incomes sustainable? firewood and timber extraction and productivity in community managed forests in Nepal," World Development, vol. 64, no. S1, pp. S113-S124, 2014.

[33] V. S. Negi, R. K. Maikhuri, and L. S. Rawat, "Non-timber forest products (NTFPs): a viable option for biodiversity conservation and livelihood enhancement in Central Himalaya," Biodiversity Conservation, vol. 20, pp. 545-559, 2010.

[34] M. Sundriyal and R. C. Sundriyal, "Underutilized edible plants of the Sikkim Himalaya: need for domestication," Current Science, vol. 85, no. 6, pp. 731-736, 2003.

[35] D. N. Tiwari, Report of the Task Force on Conservation and Sustainable Use of Medicinal Plants, Government of India Planning Commission, New Delhi, India, 2000.

[36] S. Rayamajhi, Forest dependency, livelihoods and conservation of high altitude forests in Nepal, Ph.D. Thesis, University of Copenhagen, Copenhagen, Denmark, 2010. 